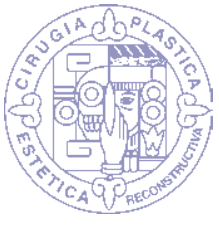

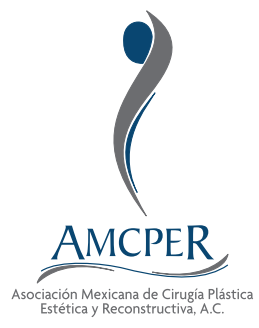

\title{
Reconstrucción de gran defecto del labio superior con colgajo musculocutáneo
}

\author{
Reconstruction of a big defect of the upper \\ lip with musculocutaneous flap
Dr. Alejandro Hurtado-Guzmán, ${ }^{*}$ Dr. Felipe Muñoz, Dra. Ángela María Londoño-García*,

\footnotetext{
Palabras clave: Labio superior, reconstrucción, colgajo musculocutáneo, orbicularis oris.

Keywords: Upper lip, reconstruction, musculocutaneous flap, orbicularis oris.
}

\section{RESUMEN}

Los defectos grandes del labio superior implican la reconstrucción tanto funcional como estética de la unidad anatómica. A lo largo del tiempo se han propuesto diferentes alternativas como colgajos locales y colgajos libres. Los cirujanos escogen entre estas alternativas dependiendo de sus indicaciones, ventajas y experiencia. Se presenta la reconstrucción de un gran defecto en el labio superior utilizando un colgajo musculocutáneo de orbicularis oris con buen resultado estético y funcional

\section{ABSTRACT}

Large defects of the upper lip involve both aesthetic and functional reconstruction of the anatomical unit. Over time, different alternatives have been proposed, such as local flaps and free flaps. Surgeons choose between these alternatives depending on their indications, advantages and experience. We present the reconstruction of a large defect in the upper lip using an orbicularis oris musculocutaneous flap, with good functional and aesthetic result.

\section{INTRODUCCIÓN}

L os defectos del labio superior son un $\_$desafío para el cirujano plástico. Aunque existen muchas técnicas de reconstrucción, no se ha propuesto ningún método específico para el cierre de grandes defectos; ${ }^{1}$ no obstante, la literatura se ha enfocado más en la reconstrucción del labio inferior por la mayor incidencia de carcinomas escamocelulares y por ser un sitio más frecuente de defectos postraumáticos. $^{2}$

Los labios son una importante unidad cosmética que además intervienen en funciones como alimentación y habla; es por esto que los principios de la reconstrucción del labio superior sean reparar las subunidades alteradas, restaurar el movimiento y sensibilidad, conservar la continencia oral y minimizar la desfiguración. ${ }^{3,4}$

En 1998, Yotsuyanagi y colaboradores ${ }^{5}$ describieron el uso de colgajos musculocutá- neos nasolabiales para la reconstrucción de defectos mayores a la mitad del labio superior, utilizando el músculo orbicularis oris del área adyacente al defecto. Esta técnica busca conservar satisfactoriamente las funciones de esfínter y apertura oral, con un resultado estético satisfactorio. ${ }^{5}$

Se presenta un caso que requirió el uso de un colgajo musculocutáneo nasolabial de orbicularis oris utilizando la técnica descrita por Yotsuyanagi para la reconstrucción de un gran defecto del labio superior luego de la resección de un tumor maligno de piel.

\section{CASO CLÍNICO}

Paciente del sexo masculino de 42 años que consultó por la aparición de una lesión en el hemilabio superior derecho de un año de evolución aproximadamente (Figura 1). La biopsia de piel reportó carcinoma basocelular de tipo nodular (Figura 2). 

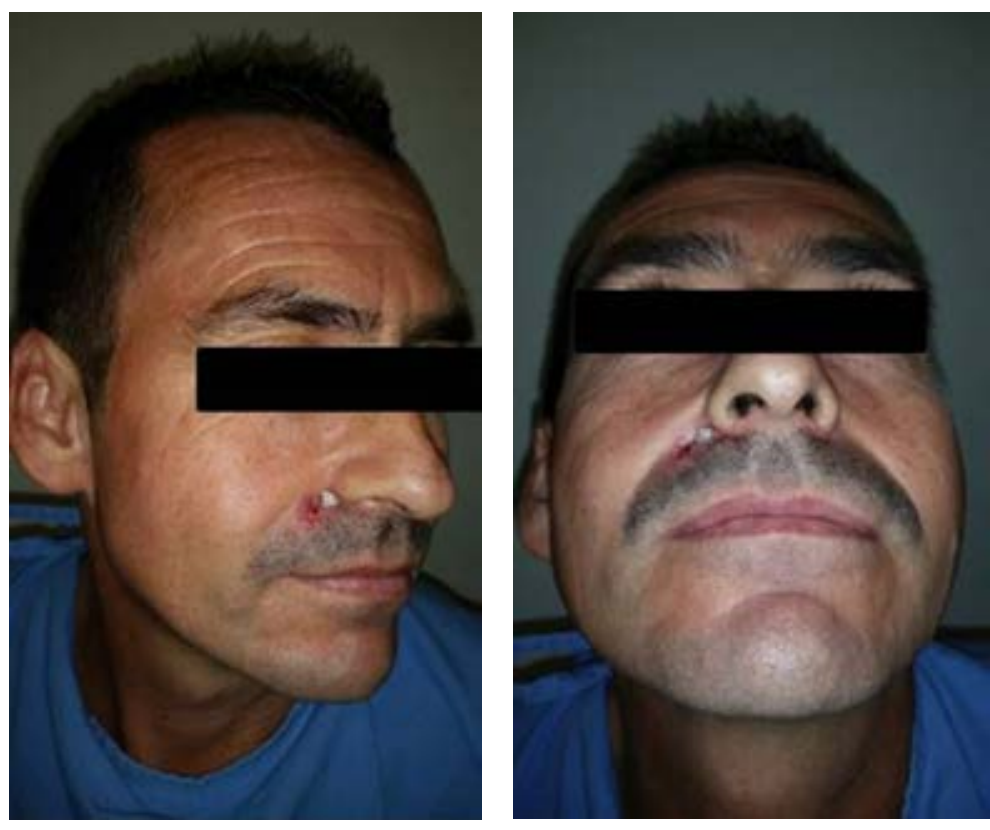

Figura 1: Placa eritematosa mal definida con una úlcera central de $1 \mathrm{~cm}$ de diámetro.

La intervención se llevó a cabo bajo anestesia general y por medio de cirugía micrográfica de Mohs se realizó la resección del tumor hasta obtener bordes libres, dejando un defecto de aproximadamente $3.0 \times 3.5 \mathrm{~cm}$, que comprometía la mitad del labio superior y parte de la mejilla infraorbitaria. Después se diseñó el colgajo teniendo en cuenta que la isla de piel estuviera sobre el territorio de la arteria facial en la región nasolabial. Con el molde listo se incidió hasta el plano subcutáneo hasta completar la liberación del colgajo, teniendo cuidado de no lesionar la porción proximal y distal de los vasos faciales. Se levantó el colgajo que incluía el orbicularis oris para transponerlo hacia la línea media y así cubrir el defecto. Por último, se afrontó por planos con sutura absorbible en el plano muscular y con sutura no absorbible monofilamento en la piel (Figura 3).

El paciente fue citado una semana después para revisar la vitalidad y función del colgajo. Se encontró al paciente en buenas condiciones generales, con el colgajo viable sin signos de infección, tolerando adecuadamente la ingesta de alimentos y la función de esfínter conservada. Ese mismo día se retiraron los puntos de sutura y se citó para nuevos controles periódi- cos cada tres meses el primer año. Al cabo de un año de la cirugía se observó una adecuada cicatrización, sin signos clínicos de recidiva tumoral y con la función del orbicularis oris conservada (Figura 4).

\section{DISCUSIÓN}

La corrección de grandes defectos del labio superior es un reto, ya que no sólo requiere buenos resultados estéticos, también debe lograr preservar las funciones que éste tiene en la masticación y el habla, teniendo en cuenta que la anatomía es más compleja con las subunidades y para un resultado ideal se debe seguir el principio de la subunidad. ${ }^{6}$

Los defectos en los labios se pueden clasificar en general como pequeños, medianos o grandes. Los pequeños defectos son aquellos que se pueden corregir con un cierre primario y no requieren un colgajo. Los defectos grandes son aquellos que son demasiado amplios para reparar con un único colgajo local y requieren una reconstrucción por etapas o un colgajo de tejido distante. Cuando se ha perdido gran parte de una subunidad, es mejor reemplazarla en su totalidad, en lugar de simplemente colocar un parche sobre el defecto. ${ }^{7}$ Para grandes defectos del labio superior, este tipo de reconstrucción provee un resultado estético satisfactorio y la preservación de la función de esfínter del orbicularis oris. ${ }^{2}$ La técnica descrita por Yotsuyanagi se prefiere en defectos de gran

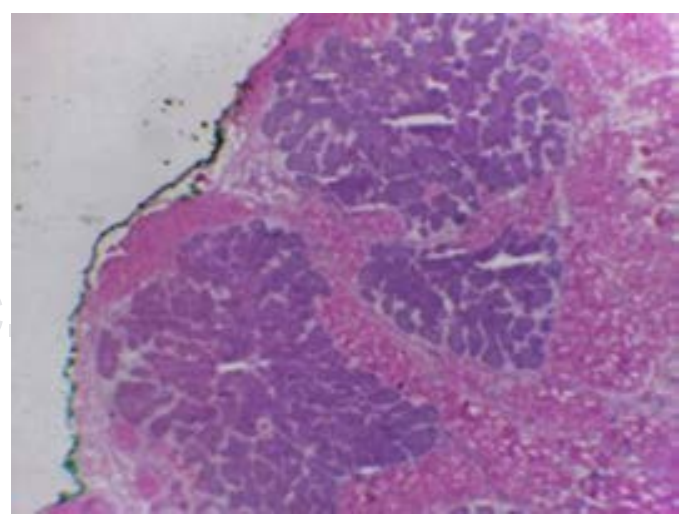

Figura 2: Nidos de células basaloides islas tumorales en una configuración nodular dentro de la dermis. 

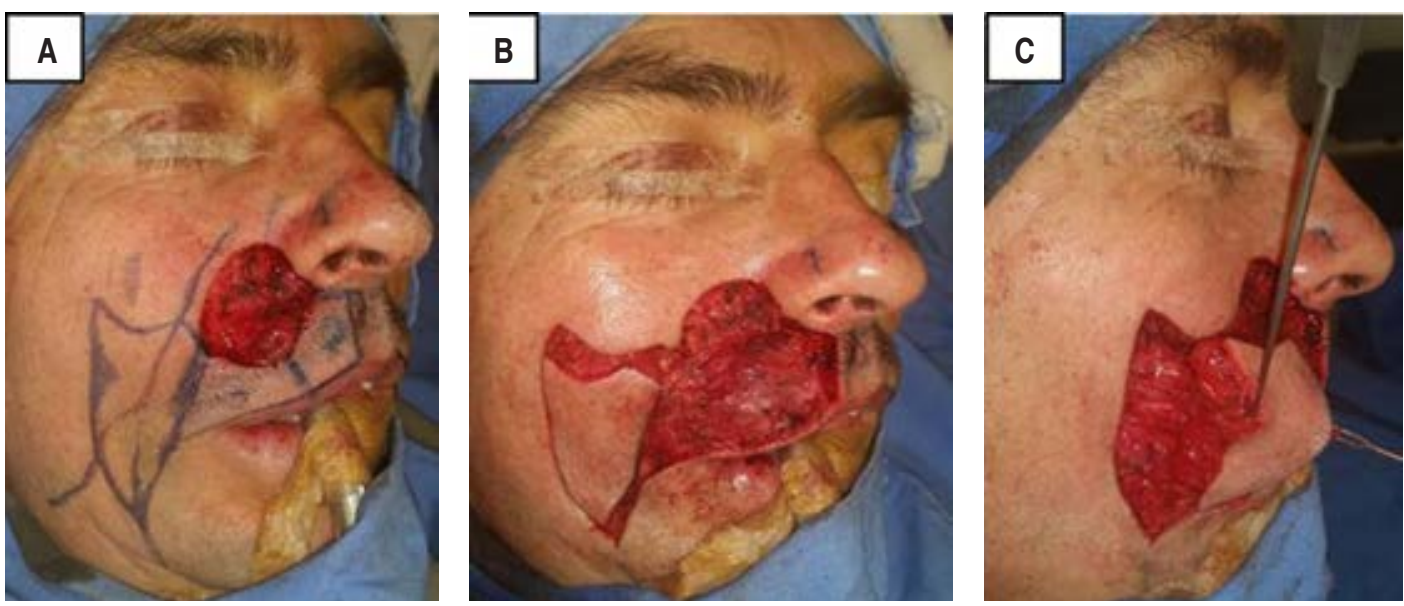

Figura 3:

A) Diseño del colgajo y marcación de la arteria facial. B) Liberación del colgajo. C) Levantamiento del colgajo. D) Transposición del colgajo. E) Corrección de bordes y cierre por planos.
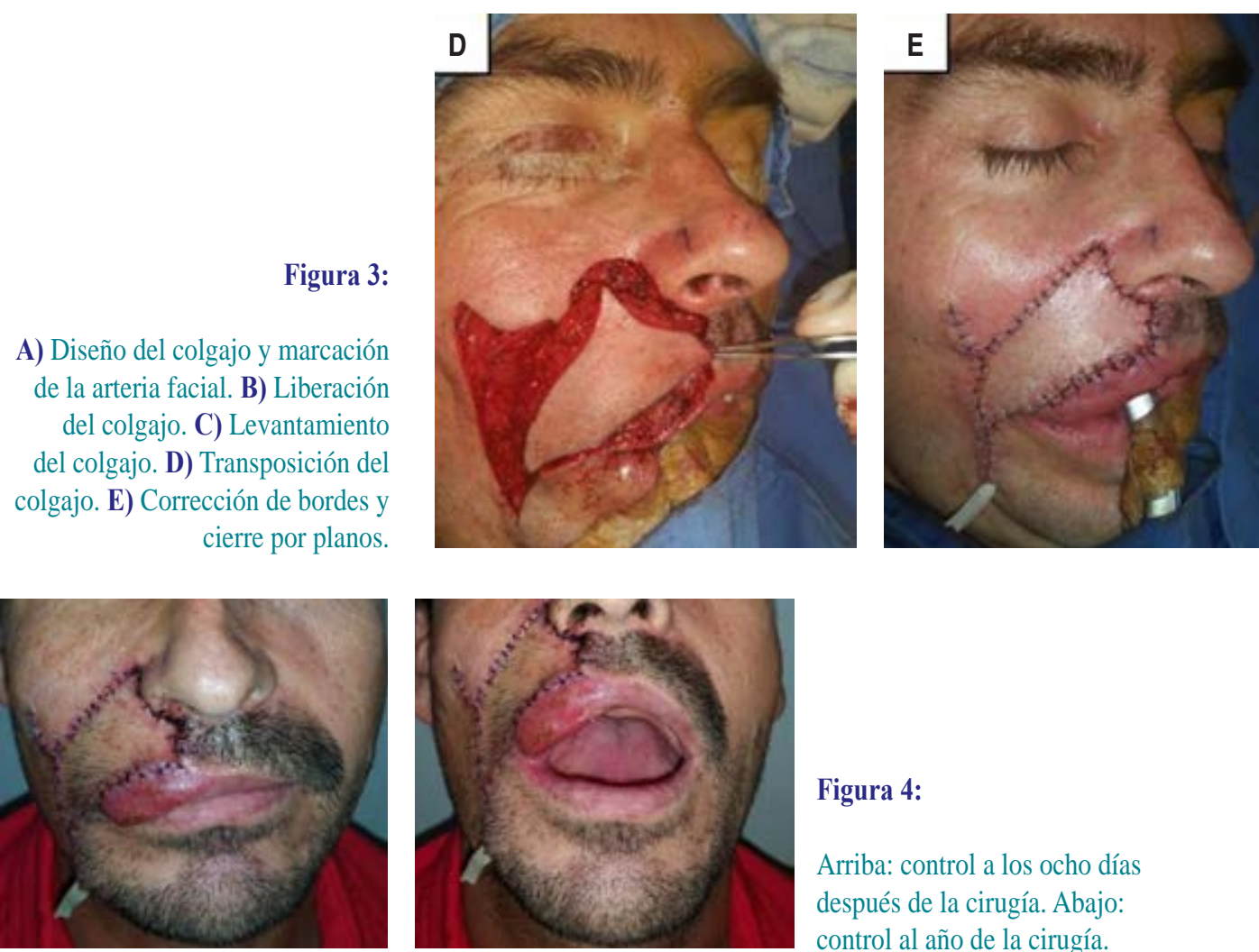

Figura 4:

Arriba: control a los ocho días después de la cirugía. Abajo: control al año de la cirugía.
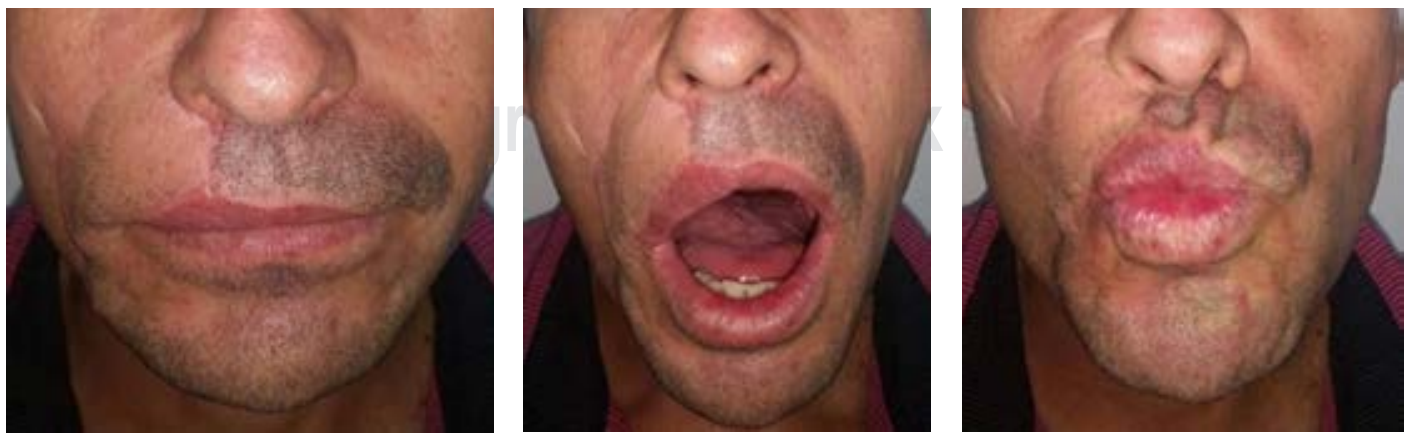
tamaño, incluso si es mayor que la mitad del labio superior, donde se conserva el ángulo de la boca. Es una opción en pacientes mayores que utilizan prótesis dentales, ya que esta reconstrucción preserva el tamaño de la boca. ${ }^{5}$

Los colgajos de avance en isla del pedículo subcutáneo son muy útiles para la reparación de los defectos cutáneos laterales del labio superior. El colgajo se libera de sus uniones del músculo orbicular cerca de la comisura y se basa sólo en la abundante grasa subcutánea lateral al ángulo de la boca. Si el defecto es mayor de $2 \mathrm{~cm}$, los resultados se mejoran al extirpar el labio restante lateral al borde medial del defecto. El resultado es una cicatriz menos visible que sigue la línea natural del pliegue melolabial hasta una posición lateral al ala nasal. ${ }^{8}$

\section{CONCLUSIONES}

El colgajo miocutáneo nasolabial con ayuda del orbicularis oris es una buena opción para reconstruir grandes defectos del labio superior; brinda beneficios cosméticos y preserva la función de apertura y esfínter de la boca con un resultado estético adecuado.

\section{REFERENCIAS}

1. Burget GC, Hsiao YC. Nasolabial rotation flaps based on the upper lateral lip subunit for superficial and large defects of the upper lateral lip. Plast Reconstr Surg 2012; 130 (3): 556-560.

2. Luce EA. Upper lip reconstruction. Plast Reconstr Surg 2017; 12. doi: 10.1097/PRS.0000000000003400.

3. Martin TJ, Zhang Y, Rhee JS. Options for upper lip reconstruction: a survey-based analysis. Dermatol Surg 2008; 34 (12): 1652-1658.

4. Cardona González MA, Chaparro Aranguren JE. Reconstrucción total de labio superior con colgajo musculocutáneo nasolabial de orbicularis oris bilateral la semana. Rev Colomb Cancerol 2013; 17 (4): 158-161.

5. Yotsuyanagi T, Yokoi K, Urushidate S, Sawada Y. Functional and aesthetic reconstruction using a nasolabial orbicularis oris myocutaneous flap for large defects of the upper lip. Plast Reconstr Surg 1998; 101 (6): 1624-1629.

6. Ishii LE, Byrne PJ. Lip reconstruction. Facial Plast Surg Clin North Am 2009; 17 (3): 445-453.

7. Sarifakioğlu N, Aslan G, Terzloğlu A, Ateş L. New technique of one-stage reconstruction of a large full-thickness defect in the upper lip: bilateral reverse composite nasolabial flap. Ann Plast Surg 2002; 49 (2): 207-210.

8. Pepper JP, Baker SR. Local flaps: cheek and lip reconstruction. JAMA Facial Plast Surg 2013; 15 (5): 374-382.

\section{Correspondencia:}

\section{Dr. Alejandro Hurtado-Guzmán}

Torre Médica C.C. El Tesoro, consultorio 731, Carrera 25A Núm. 1A Sur-45,

Medellín, Colombia.

E-mail: alejohur@hotmail.com 